\title{
CORRESPONDENCE
}

\section{Assessment of safety and feasibility of human allogeneic adipose-derived mesenchymal stem cells in a pediatric patient}

Pediatric Research (2018) 84:575-577;

https://doi.org/10.1038/s41390-018-0042-8

To the Editor: Human adipose-derived mesenchymal stem cells (hAd-MSC) have been the subject of many investigations and has attracted significant attentions as to their potential therapeutic applications. ${ }^{1-3}$ Ad-MSC possess pleuripotential properties capable of differentiating into several types of cells such as adipocyte, myocyte, chondrocyte, and osteocyte. ${ }^{4}$ Intravenous administration of Ad-MSC showed accumulation mainly in the lungs, and also in the heart, liver, and brain. ${ }^{5}$ MSC has been used to help alleviate various disease conditions. Long-term follow-up of patients with ischemic stroke after MSC administration showed some improvement with no adverse effects after 5 years. ${ }^{6}$

On the other hand, cerebral palsy (CP) is a heterogenic disability of varied etiology. CP is a severe disabling disease with a worldwide incidence of two to three per 1000 live births. ${ }^{7} \mathrm{CP}$ involves impaired movement, muscle function, and cognitive functioning, with effects ranging from mild to severe. No biological intervention has been effective for $\mathrm{CP}$, and the standard approach is limited to supportive management. Currently, stem cell-based strategies have garnered attention due to their ability to stimulate neuroregeneration and neuroprotection in CP..$^{8-11}$

Allogenic umbilical cord blood MSCs have been used in spastictype CP in eight pairs of identical twins ${ }^{9}$ and in studies with $80,{ }^{10}$ $17^{8}$ and $47^{11}$ patients with CP. No serious adverse effects were reported. Also, a recent study ${ }^{12}$ administered multiple intrathecal and intravenous infusions of allogenic bone marrow-derived MSCs to three children with spinal muscular atrophy type 1, and observed improvements in the patients' physical functioning (PF). No adverse effects on follow-up for at least 3 years was observed and the treatment was considered safe and effective.

Here we assessed the safety and efficacy of allogeneic hAd-MSC administration in a pediatric patient with CP. To our knowledge, this is the first report on the use of allogeneic human adipocyte MSC in pediatric patients with CP.

The patient was a 7-year-old boy with a history of cerebral infarction at 2 years of age due to hemolytic uremic syndrome following Escherichia coli 0-157 infection who visited the Sun Field Clinic in June 2012. The right thalamus putamen zone was involved in the infarcted area and the patient had developed $C P$ on the left side of body, with facial nerve disorder. He had been given multiple courses of steroids since 2007. On admission, he had left arm stiff muscles, shoulder pain, palm dermatitis, and gait disturbance. Because of the lack of response to steroid therapy and in the light of encouraging results from MSC administrations in improving CP manifestations, we took advantage of allogeneic hAd-MSC administration.

Adipose tissue was harvested from the peri-umbilical area of the patient's mother using a simple lipoaspiration method under local anesthesia and used for hAd-MSC isolation. A total amount of $22 \mathrm{~g}$ of fatty tissue was obtained. Written informed consent and consent to publish were obtained from the patient's guardian and donor before performing the procedures. All procedures were performed following the ethical policies of the Sun Field Clinic in Tokyo, Japan and were approved by the Sun Field Clinic Internal Review Board.

Allogeneic hAd-MSCs were isolated using a combination of enzymatic digestion and centrifugation. In brief, the fat tissue was digested using collagenase enzyme $(300 \mathrm{U} / \mathrm{ml})$ at $37^{\circ} \mathrm{C}$ for $2 \mathrm{~h}$ and reconstituted in Hank's balanced salt solution at $37^{\circ} \mathrm{C}$ for $60 \mathrm{~min}$. The collagenase was then inactivated in serum-free proliferation medium (sfDOT; BioMimetics Sympathies, Inc.) supplemented with L-glutamine $(2 \mathrm{mM})$, penicillin $(100 \mathrm{U} / \mathrm{ml})$, and streptomycin $(100 \mu \mathrm{g} / \mathrm{ml}){ }^{13}$ After centrifugation at $400 \times g$ for $5 \mathrm{~min}$ and filtration through a $100 \mu \mathrm{m}$ filter (Nylon cell strainer; Becton Dickinson, Franklin Lakes, NJ, USA), the sample was centrifuged at $400 \times g$ for $5 \mathrm{~min}$ and resuspended in the same serum-free proliferation medium. This stromal vascular fraction was referred to as passage zero. Cell cultures originated from this specimen was incubated for $48 \mathrm{~h}$ at $37^{\circ} \mathrm{C}$ in a $5 \% \mathrm{CO}_{2}$ incubator, washed with phosphate-buffered saline, and after sequential trypsin treatments, they were maintained in the serum-free proliferation media up to passage three. The hAMSCs were suspended in a cryopreservation solution at a cell concentration of $1 \times 10^{7}$ cells per $\mathrm{ml}$ and immediately cryopreserved in liquid nitrogen. A mean of $16 \pm 3$ days was required for the production of hAd-MSC and the time needed for thawing the cryopreserved cells and making it ready for administration was about $1 \mathrm{~h}$.

Selection of hAd-MSC was based on their capacity to adhere to the surface of plastic culture flasks. Using flow cytometric analysis, the hAd-MSCs were confirmed as stem cells mainly by a positive expression of CD73 and CD90 and negative expression of CD31 and CD45. Cell viability and survival were determined by trypan blue staining and exclusion test and the rate was set at $>80 \%$. In addition, the hAd-MSC could differentiate to fat, bone, and cartilage tissue cells, and inhibit the proliferation of T cells that are the same characteristics as MSCs.

The patient received allogeneic human hAd-MSC administration at indicated doses and followed up at least for 12 months. The patient's clinical and laboratory records were reviewed with special reference to treatment safety, feasibility, and efficacy issues. Patient's vital signs and physical examination had been checked systematically after each treatment. Safety and feasibility were evaluated using several measures, as described elsewhere. ${ }^{14}$

We also assessed the efficacy after administration by observing changes in the clinical manifestations of the various disorders at each patient visit $(1,2,3,6$, and 11 months) using the Short Form 8 (SF-8) Health Survey Quality of Life (QoL) questionnaire developed by QualityMetric Incorporated (Lincoln, RI, USA) and the Gross Motor Function Classification System (GMFCS). The scoring evaluation was done without the knowledge of the previous scores. The SF-8 generates a health profile with eight discrete scores describing the health-related QoL, which are summarized as continuous physical component summary (PCS) and mental component summary (MCS) scores. These summary scales (PCS and MCS) have been normalized for the Japanese population. ${ }^{15}$ 


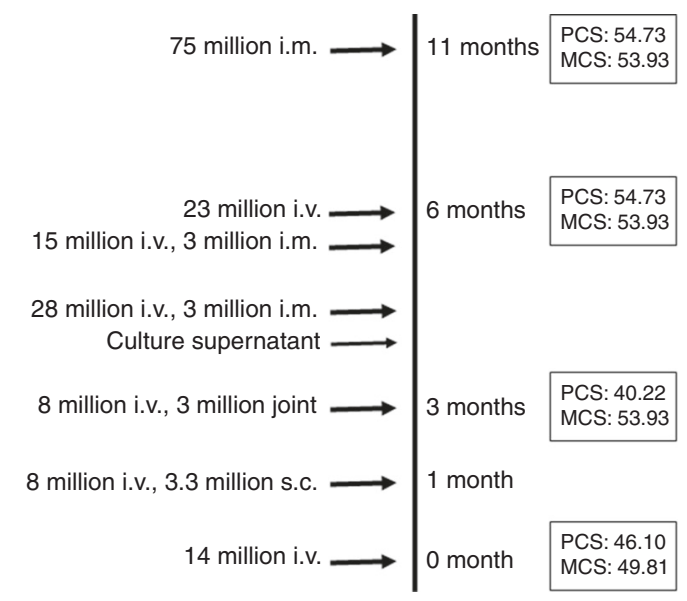

Fig. 1 The changes in physical component summary and mental component summary score from baseline to the 11 months followup after allogeneic human adipose-derived mesenchymal cells transfusions are shown. At final 12 months follow-up, the PCS and MCS scores were stable between 5 and 12 months after treatment. i.v. intravenously, i.m. intramuscularly, s.c. subcutaneously, MCS Mental component summary, PCS physical component summary

Briefly, the questionnaire has the practical advantage of being brief, while yielding scores that are directly comparable to the eight scores produced by the standard SF-36 questionnaires. In order to calculate these comparable scores, the SF-8 survey first measures the following eight ordinal items: general health (SF8GH), PF (SF-8PF), role physical (RP) (SF-8RP), bodily pain (SF-8BP), vitality (SF-8VT), social functioning (SF-8SF), mental health (SF$8 \mathrm{MH}$ ), and emotional roles (SF-8RE). Scale means based on the same standard metrics as the SF-36 are assigned to ordinal response, creating a continuous outcome for each of the eight items. Regression coefficient weights are assigned to each item to produce a PCS and MCS score using Japanese healthy control data. ${ }^{16}$ The normalized scores for PCS and MCS in the Japanese population are 49.84 and 50.09, respectively, in 2007.

Developmental screening tests and developmental and medical examinations before and after the hAd-MSC administration were used to assess improvements in the patient's clinical condition. Patient's vital signs and physical examination was systematically checked after each treatment. Except for a slight fever after the second administration of hAd-MSC that resolved quickly no other adverse effects or complications were observed.

Figure 1 shows the changes in PCS and MCS score from baseline to the 11-month follow-up. The PCS score decreased by 5.88 at 1 month after hAd-MSC administration, and then gradually increased by 14.51 during the subsequent 4 months. Ultimately, the PCS score had increased by 8.63 at the 5 months follow-up. Of the eight original variables on the SF-8 Health Survey QoL (general health, PF, RP], bodily pain, vitality, social functioning, mental health, and emotional roles), two (PF and RP) were lower at 1 month after hAd-MSC administration compared with before treatment. Four months later, the patient had higher scores for all eight items, including PF and RP, compared with scores before treatment. MCS score increased by 4.12 at 1 month after hAd-MSC administration, and there was no change in MCS score in the subsequent 4 months. At the 5 months follow-up, the MCS score was 4.12 higher than before treatment. At 11 months after the hAd-MSC treatment, the patient maintained his recovery with no significant change. He received $75 \times 106$ hAd-MSCs intramuscularly in the left upper arm. At final 12 months followup, the PCS and MCS scores were stable between 5 and 12 months after treatment. The GMFCS classification of the patient showed improvement from class 2 before treatment to class 1 after treatment.
In this report, we demonstrated safety and feasibility of allogeneic hAd-MSC in patients suffering from CP. There were no adverse effects, and significant improvements in the clinical manifestations and QoL scores were observed. The hAdMSC administration had positive effects on both physical and mental health. Remarkably, the PCS, MCS, and GMFCS scores improved. In a recent study on patients with spastic $C P$, bone marrow MSCs proved to be safe and feasible and could improve gross motor and fine motor functions significantly during a 12-month follow-up. ${ }^{17}$

Our patient had developmental and physical disabilities resulting from $C P$ and was qualified to benefit from the effects of hAd-MSC administration. The advantages of hAd-MSC over other types of hMSC lie in the fact that they are easily obtainable from subcutaneous fat tissue with minor pain and morbidity, express low immunogenicity, and can differentiate not only into cells of mesodermal origin (adipocytes, osteoblasts, chondrocytes, and muscle cells) but also cells of ectodermal and endodermal origins (epidermal and neuronal cells). They are also free from ethical issues and risk of tumor formation as associated with embryonic MSC. In addition, the optimal treatment schedule, dose, and route of administration for hAd-MSC has not yet been established and may depend on the disease entity, organs involved, symptoms, and response to therapy, preferably considering targeted and regional delivery. Basically, a dose of $1 \times 10^{6}$ cells per $\mathrm{kg}$ body weight has been used in several studies. In our patient, various doses and routes of administration were employed depending on the patient's condition during the course of therapy. Although this is a single case, however, the encouraging results add support to the safety and feasibility of allogeneic hAd-MSC application and suggest further investigations in pediatric patients with CP.

\section{ADDITIONAL INFORMATION}

Competing interests: The authors declare no competing interests.

Atsuyuki Hirano ${ }^{1}$, Masayuki Sano ${ }^{1}$, Naoki Urushihata ${ }^{2}$, Hideki Tanemura ${ }^{2}$, Katsuyuki Oki ${ }^{2}$ and Emi Suzaki ${ }^{2}$ ${ }^{1}$ Cosmetics and Regenerative Medicine, Sun Field Clinic, Aomi, Kotoku, Tokyo, Japan and 'BioMimetics Sympathies Inc., 2-4-32 Aomi, Koto-ku, Tokyo, Japan Correspondence: Atsuyuki Hirano (a-hirano@renais-clinic.com)

\section{REFERENCES}

1. Gimble, J. M., Katz, A. J. \& Bunnell, B. A. Adipose-derived stem cells for regenerative medicine. Circ. Res. 100, 1249-1260 (2007).

2. Vonk, L. A., de Windt, T. S., Slaper-Cortenbach, I. C. \& Saris, D. B. Autologous, allogeneic, induced pluripotent stem cell or a combination stem cell therapy? Where are we headed in cartilage repair and why: a concise review. Stem Cell Res. Ther. 6, 94 (2015).

3. Bajek, A. et al. Adipose-derived stem cells as a tool in cell-based therapies. Arch. Immunol. Ther. Exp. 64, 443-454 (2016).

4. Zuk, P. A. et al. Multilineage cells from human adipose tissue: Implications for cell-based therapies. Tissue Eng. 7, 211-228 (2001).

5. Katz, A. J. \& Mericli, A. F. in Principles of Regenerative Medicine (eds Atala, A., Lanza, R., Thomson, J. A. \& Nerem, R.) 365-381 (Academic Press, San Diego, 2011)

6. Lee, J. S. et al. A long-term follow-up study of intravenous autologous mesenchymal stem cell transplantation in patients with ischemic stroke. Stem Cells 28, 1099-1106 (2010).

7. Mutch, L., Alberman, E., Hagberg, B., Kodama, K. \& Perat, M. V. Cerebral palsy epidemiology: where are we now and where are we going? Dev. Med. Child. Neurol. 34, 547-551 (1992).

8. Kang, M. et al. Involvement of immune responses in the efficacy of cord blood cell therapy for cerebral palsy. Stem Cells Dev. 24, 2259-2268 (2015).

9. Wang, X. et al. Effect of umbilical cord mesenchymal stromal cells on motor functions of identical twins with cerebral palsy: pilot study on the correlation of efficacy and hereditary factors. Cytotherapy 17, 224-231 (2015). 
10. Romanov, Y. A. et al. Human allogeneic ABO/Rh-identical umbilical cord blood cells in the treatment of juvenile patients with cerebral palsy. Cytotherapy 17 969-978 (2015).

11. Feng, M. et al. Safety of allogeneic umbilical cord blood stem cells therapy in patients with severe cerebral palsy: a retrospective study. Stem Cells Int. 2015, 325652 (2015).

12. Villanova, M. \& Bach, J. R. Allogeneic mesenchymal stem cell therapy outcomes for three patients with spinal muscular atrophy type 1. Am. J. Phys. Med. Rehabil. 94, 410-415 (2015)

13. Aso, K. et al. Adipose-derived mesenchymal stem cells restore impaired mucosal immune responses in aged mice. PLOS ONE 11, e0148185 (2016).
14. Vériter, S. et al. Human adipose-derived mesenchymal stem cells in cell therapy: safety and feasibility in different "hospital exemption" clinical applications. PLoS ONE 10, e0139566 (2015).

15. Ware, J.E., Kosinski, M., Dewey, J.E., Gandek, B. in How to Score and Interpret SingleItem Health Status Measures: A Manual for Users of the SF-8 Health Survey (QualityMetric Incorporated, Lincoln, 2001).

16. Fukuhara, S. \& Suzukamo, Y. Manual of the SF-8 Japanese Version (Institute for Health Outcomes and Process Evaluation Research, Kyoto, 2004).

17. Liu, X. et al. Comparative analysis of curative effect of bone marrow mesenchymal stem cell and bone marrow mononuclear cell transplantation for spastic cerebral palsy. J. Transl. Med. 15, 48 (2017). 